\title{
Molecular spin echoes; multiple magnetic coherences in molecule surface scattering experiments.
}

\author{
Helen Chadwick, ${ }^{1}$ Yosef Alkoby, ${ }^{1}$ Joshua T. Cantin, ${ }^{1}$ Dennis \\ Lindebaum, ${ }^{1}$ Oded Godsi, ${ }^{2}$ Tsofar Maniv ${ }^{2}$ and Gil Alexandrowicz ${ }^{1}$ \\ ${ }^{1}$ Department of Chemistry, College of Science, \\ Swansea University, Swansea, SA2 8PP, UK \\ ${ }^{2}$ Schulich Faculty of Chemistry, Technion Israel Institute \\ of Technology, 32000 Technion City, Haifa, Israel
}

(Dated: November 30, 2020)

\begin{abstract}
In this paper we demonstrate that a molecular beam of hydrogen molecules can be magnetically manipulated to produce multiple coherences in the molecular interference pattern. Unlike spin $\frac{1}{2}$ magnetic beam experiments, i.e., neutron and helium spin echo, the nuclear and rotational magnetic moments in a molecule are strongly coupled. We show experimentally and theoretically that this coupling leads to multiple magnetic field conditions under which the magnetic moment of molecules travelling with different speeds can be coherently refocussed. We also demonstrate that these multiple coherence signals are extremely sensitive to the scattering event, opening up new possibilities for measuring molecule-surface interactions.
\end{abstract}




\section{INTRODUCTION}

Spin echo beam experiments have revolutionised the ability to study motion within the bulk and on the surface. Initially, the coherent magnetic interference phenomena was demonstrated with a cold neutron beam and applied to measure bulk dynamics [1, 2]. The neutron experiment, termed neutron spin echo, has since become a well established and widely used technique, accessing time scales which can not be studied using time of flight neutron experiments [3]. A couple of decades later, a similar measurement was shown to be possible with ${ }^{3} \mathrm{He}$ beams [4]. Helium spin echo measurements have since been used to study various surface systems which were previously inaccessible to experiments, these include studying ultra-fast diffusion of molecules on surfaces, inter-adsorbate interactions between adsorbed species, energy dissipation on surfaces, surface phonons and many other examples [5, 6]. The basic signal which lies at the heart of these experiments is the spin echo signal.

The simple and widely used classical explanation for the spin echo signal is the following: the magnetic moments of the beam particles, which pass through a magnetic field, $B_{1}$, undergo Larmor precessions and their total accumulated spin phase depends on the time spent in the field, i.e., on the particle velocity. The finite width of the velocity distribution leads to a phase spread and a loss of coherency. By passing the beam through a second magnetic field, $B_{2}$, located after the interaction with the sample, reversed precession can be achieved. As a result, the velocity spread can be refocussed and a revival of coherency can be obtained, which is termed the spin echo signal [2], in analogy with the concept of spin echoes in NMR [7]. More recent work has demonstrated that the scattering geometry of the spin echo apparatus actually leads to two spin echo conditions $B_{1}=B_{2}$ and $B_{1}=-B_{2}$, where the two are referred to as the parallel and anti-parallel spin echoes [8]. The most common use of these spin echo signals, is to measure motion within or on the sample, making use of the fact that motion, taking place during the scattering event, reduces the coherency and hence the intensity of the echo signal. In particular, it can be shown using classical, semiclassical and quantum mechanical explanations that the intensity of the spin echo signal measured while scanning the magnitude of the magnetic fields $\left|B_{1}\right|=\left|B_{2}\right|$, is proportional to the intermediate structure factor, which is the Fourier transform of the time dependent pair correlation function describing the dynamics of the sample [2, 9-11].

In this paper, we will examine how the concept of spin echoes extends to molecular beams. 
Generally speaking, the extension of atomic beam experiments to molecules leads to challenges and complexities both in terms of the technical requirements for the experiments and in terms of the interpretation of the measurements. Nevertheless, as has been demonstrated for the case of surface diffraction, bound state resonance measurements, phonon studies and many other examples, the extension of the experiments to molecular beams also leads to new intriguing insights [12-14]. Below we show that the relatively simple spin echo phenomena obtained for spin $\frac{1}{2}$ particles is replaced by a rich and complex signal, characterised by multiple coherence features with intensities which depend strongly on the scattering event, opening a new wide range of future experiments to study molecule-surface interactions.

The experiments and calculations presented in this paper were performed for a rotationally cold hydrogen molecular beam. A supersonic expansion and the large rotational constants ensure that our beam consists of the two lowest rotational states, para- $\mathrm{H}_{2}$ in $J=0$ (with a nuclear spin $I=0$ ) and ortho- $\mathrm{H}_{2}$ in $J=1$ (and $I=1$ ). The former is magnetically inert and so simply appears as a constant background in our experiments. In contrast, ortho- $\mathrm{H}_{2}$ interacts with magnetic fields. The effect of a magnetic field on a ground state ortho- $\mathrm{H}_{2}$ molecule was studied in detail by Ramsey and co-workers, leading to the development of an accurate Hamiltonian for this system [15]. There is one particularly important difference between the propagation of an ortho- $\mathrm{H}_{2}(J=1)$ molecule in a magnetic field and that of the more popular spin $\frac{1}{2}$ probes (neutrons and ${ }^{3} \mathrm{He}$ atoms): while for neutrons and helium atoms the interaction is only through the Zeeman term which couples the nuclear spin with the applied magnetic field, for the case of ortho- $\mathrm{H}_{2}$, there are additional non-negligible spin-rotation and spin-spin coupling terms, which are referred to as non-Zeeman terms, and are independent of external magnetic fields.

The existence of these additional terms raises the question of whether it is at all possible to obtain a spin echo signal in this molecular system. While reversing the magnetic field will reverse the phase evolution of the Zeeman terms, it will not affect the evolution taking place due to the field independent terms. Hence, it is not immediately clear how one can regain the phase coherency of molecules which propagate through the beam line with different speeds, and obtain a spin echo signal. First experimental evidence, measured for $\mathrm{H}_{2}$ scattering from a copper surface showed that the spin coherency can be partially regained at the conventional spin echo conditions (equal and opposite magnetic fields) [16]. Below we will show that this is just one of many magnetic field conditions where spin coherency can be regained and spin 
echo like features can be measured.

\section{EXPERIMENTAL}

The apparatus used to measure the multiple echo phenomena is shown schematically in Fig. 1. The supersonic molecular beam is produced in the source chamber from a nozzle held at a temperature of $100 \mathrm{~K}$, and then enters the polariser, a hexapole magnet field [17] with a transmission probability that depends on the rotational $\left(m_{J}\right)$ and nuclear spin $\left(m_{I}\right)$ projection state. As a consequence, only a fraction of state selected molecules continue along the beamline, whereas other states are deflected out of the molecular beam. Due to the strong magnetic field gradients within the hexapole, superposition states undergo destructive interference [18] and we are left with pure eigen-states along the $z$ quantisation axis (defined by the direction of a dipole element at the end of the hexapole). We denote the eigen-states as $\psi_{i}$, where the index $i$ includes the nine different $m_{I}, m_{J}$ states of the ortho- $\mathrm{H}_{2}$ $J=1$ molecules. In an ideal experiment, the particles would then propagate through a zero magnetic field region where the wave-function evolves due to the non-Zeeman terms in the Hamiltonian. However, there are small residual magnetic fields in part of this region which are shown in the real magnetic field profile of the first arm given in Fig. S1 in the Supplementary Information. The beam then enters the first electromagnet $\left(B_{1}\right)$, which produces a field which is aligned antiparallel to the beam propagation axis (i.e., along $-x$ ). Within this region the wave-function evolves under both the Zeeman and non-Zeeman terms of the Hamiltonian. After exiting $B_{1}$ the beam enters a magnetically shielded scattering chamber where the beam scatters from the surface. Within this region the wave-function propagation is affected by the flight through the zero field regions before and after the sample and the scattering event itself. The scattered particles which enter the second arm of the instrument, pass through a second electromagnet $\left(B_{2}\right)$, oriented along the $-x^{\prime}$ direction (rotated by $135^{\circ}$ with respect to $-x$ ), a second zero field region, and finally are selected by a spin analyser (another hexapole magnet [19]). The analyser transmits them towards the particle detector with a probability which depends on their projection along the $z^{\prime}$ quantisation axis (see Fig. 1). One feature of the apparatus which is different from other spin echo machines and is particularly relevant for this study, is that the two electro-magnets are independently controlled by high stability power supplies (Danfysik - 854). This allows 


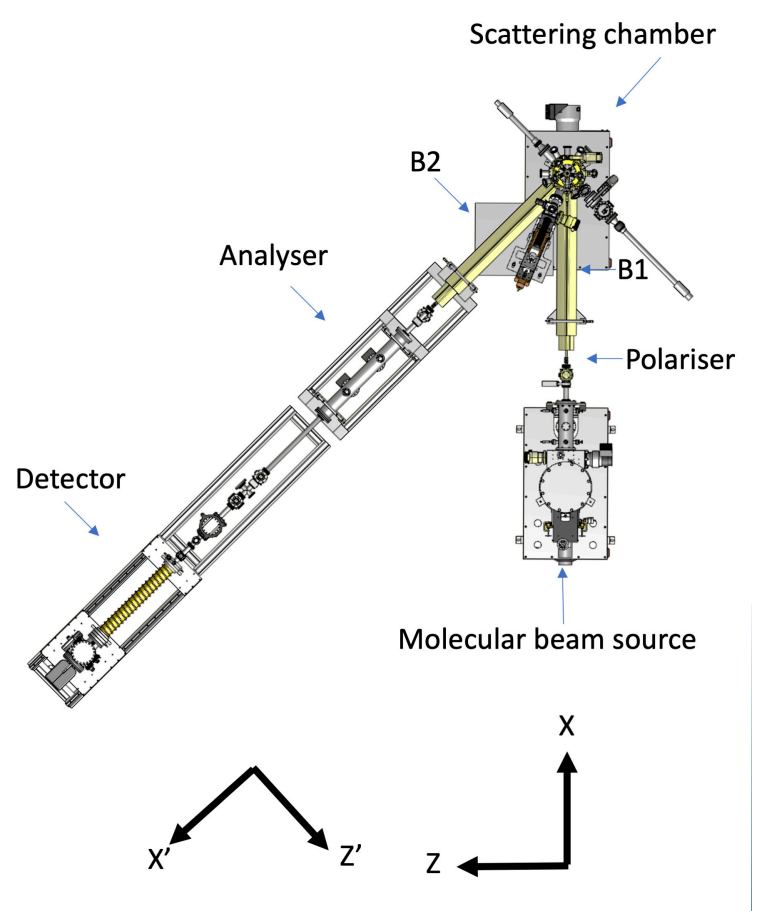

FIG. 1: Schematic of the experimental apparatus, showing the main elements of the experiment and the axes definitions used in the text.

the current in the two electro-magnets to be scanned independently and is essential for measuring coherences far away from the traditional spin echo condition $\left|B_{1}\right|=\left|B_{2}\right|$.

The molecular beam apparatus described above can produce detailed insight into the scattering event when used in conditions which circumvent the need for velocity spread refocussing. One way of reducing the effect of the velocity spread is to perform diffraction scattering measurements, using the crystal as a monochromator. Due to the high angular resolution of the instrument, the spread of velocities contributing to the diffraction peak signal is dramatically narrower, allowing the signal to be measured without the need for echo refocussing. Such experiments are capable of extracting the scattering matrix from the data, a unique observable which describes the changes in the quantum wave-functions that occur during scattering [20]. In contrast, when measuring under more general scattering conditions, including specular scattering, the full width of the velocity distribution of the beam particles contributes to the signal. In this case we need to find conditions where the velocity dependence of the magnetic moment is not important up to first order and the averaging over the beam particles results in a finite signal, i.e., the spin echo phenomena.

Panel a of Fig. 2 shows the intensity of the specularly scattered signal (incident angle 
$\left.=22.5^{\circ}\right)$ for a $\mathrm{H}_{2}$ beam directed perpendicular to the step edges of a $\mathrm{Cu}(511)$ surface at a temperature of $200 \mathrm{~K}$ as the field in the first solenoid was scanned between 0 and 600 gauss.m and the second was held fixed at 545 gauss.m. An oscillating signal, centred near the standard spin $\frac{1}{2}$ echo condition $\left|B_{1}\right|=\left|B_{2}\right|=545$ gauss $\cdot \mathrm{m}$ can be seen. Panel b focusses on this feature, showing that while the envelope of the signal is located at the standard spin echo position, the internal oscillations contain more than one frequency which reflects the complexity of the Hamiltonian in comparison to the spin $\frac{1}{2}$ case [16]. Looking at other regions of the upper panel, we see that there are a number of other echo signals which can be seen above the experimental noise, located at various $B_{1}$ values. Panels c and d focus on two of these echoes located at $B_{1}$ values which are approximately 400 and 100 gauss $\cdot \mathrm{m}$ lower. Panel e magnifies the oscillations in the signal in the region $B_{1}=0$ gauss.m. The intensity of these oscillations is stronger than the various echoes mentioned above and it is dominated by the flux-detection signal, a point we will address in more detail later.

\section{SIMULATIONS}

To better understand the phenomena and complexity of molecular spin echoes we perform calculations of the simulated signal at various magnetic field values. The calculations are based on a combination of a fully quantum treatment of the magnetic Hamiltonian and a semi-classical description of the centre of mass of the molecule [16]. In brief, the probabilities that the different $m_{I}, m_{J}$ states are transmitted through the polariser $\left(P_{\text {hex } 1}\right)$ and analyser $\left(P_{\text {hex } 2}\right)$ hexapoles are determined using semi-classical trajectory calculations. The wave-function for each individual $m_{I}, m_{J}$ state is obtained by using the magnetic Hamiltonian to propagate the wave-function through a vector-valued magnetic field profile that the molecules experience when travelling through the first arm and second arm of the machine. These profiles include both the dipole and solenoid fields as well as small residual fields that have been measured throughout the molecular beam line, and are shown in Figs. S1 and S2 in the Supplementary Information for the first and second arms respectively. This propagation is described by a $9 \mathrm{x} 9$ matrix, $U\left(B_{n}\right)$, which characterises the coherent superposition of $m_{I}, m_{J}$ states that each of the initial pure $m_{I}, m_{J}$ states evolves into during the propagation through each arm of the machine. The interaction of the molecules with the surface can be described by the scattering matrix $(S)$. This matrix could be either extracted directly 

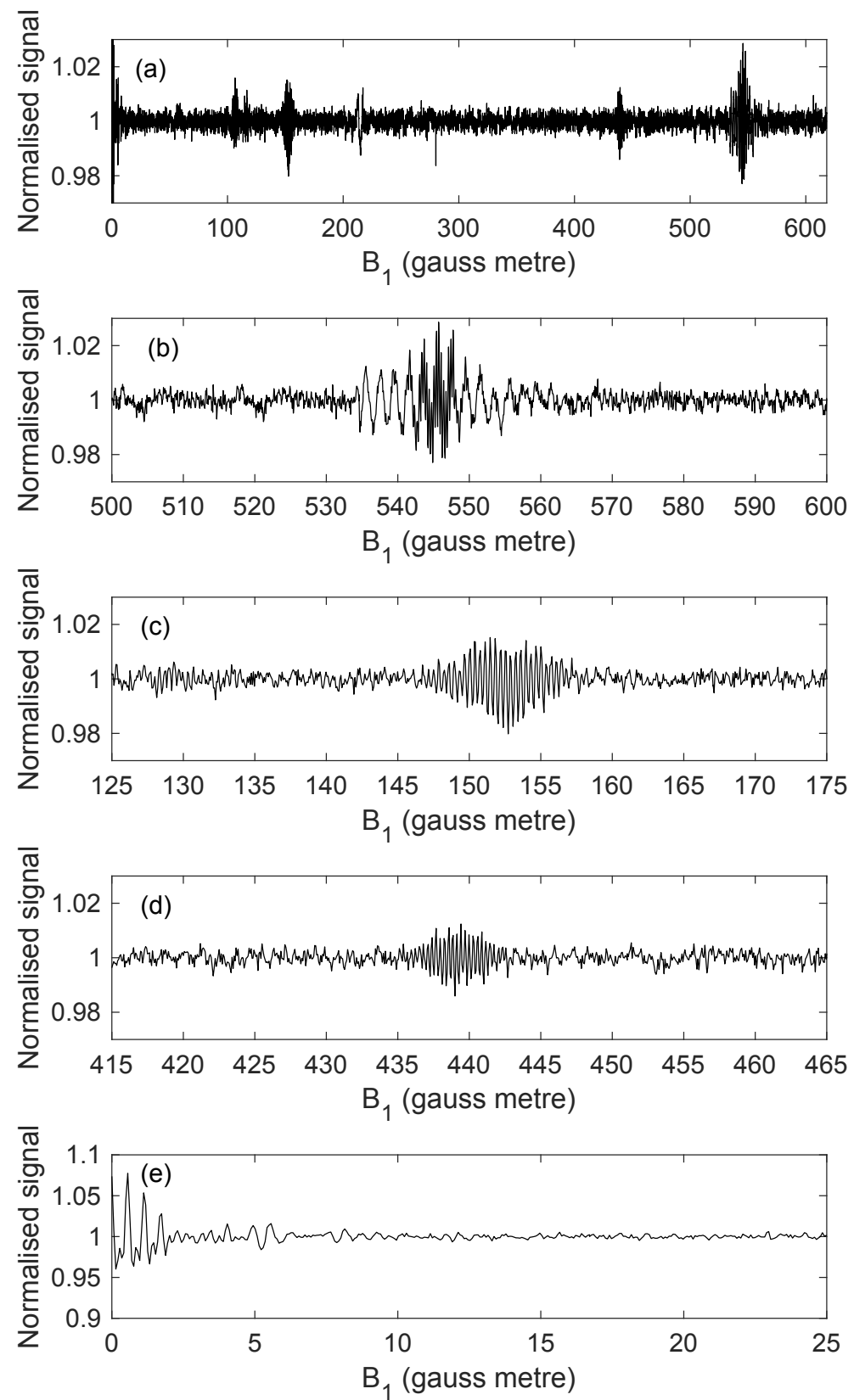

FIG. 2: Normalised detector count rate as function of the field integral strength of the first electromagnet for $\mathrm{H}_{2}$ scattering from a $\mathrm{Cu}(511)$ surface at a surface temperature of $200 \mathrm{~K}$. The second electromagnet was maintained at a fixed value of $B_{2}=545$ gauss $\cdot \mathrm{m}$. Multiple signals, reflecting a refocussing of the phase coherency can be seen in the panel (a). The remaining panels magnify some of these features. Panel (b) shows an echo centred at the traditional spin echo condition $\left|B_{1}\right|=\left|B_{2}\right|$, and panels (c) and (d) focus on echoes far from this condition. Finally panel (e) focusses on the initial oscillations dominated by the flux-detection signal as discussed later in the text. Note that these oscillations are more intense and the vertical scale is different than that of other panels to not truncate the signal. 
from the data [20], or from a scattering calculation. When analysing helium scattering experiments [12], the scattering calculations and the measurements are compared by a single number, namely the total scattered intensity in a particular direction, this value corresponds to the sum of the square of all of the elements in the scattering matrix. A more detailed description of the gas-surface collision is obtained from the full scattering matrix, where each element represents the corresponding change in the wave-function for that particular scattering channel. In the case that there are no magnetic interactions during scattering, as considered here, the $S$ matrix would simply be proportional to an identity matrix for ${ }^{3} \mathrm{He}$ scattering, as it would not be possible to change the $m_{I}$ state during the collision. In the case of $\mathrm{H}_{2}$, the $m_{I}$ state will not change due to the lack of magnetic interactions, and as the timescale of the collision is short compared to the time spin-rotation effects would become significant. However, the $m_{J}$ state can change, meaning we can simply use a $3 \times 3$ matrix to quantify how the amplitudes and phases of the wave-function changes. This matrix can then be expanded to a 9x9 matrix where each $m_{J}$ to $m_{J}$ element is the same for all values of $m_{I}$. As $m_{J}$ state changing collisions can occur, the scattering matrix is not necessarily diagonal. It will also rarely be unitary, as in most cases there are several different channels that the $\mathrm{H}_{2}$ can scatter into, meaning that all the flux is unlikely to be scattered into the observed channel.

The intensity, $I$, of the scattered signal for a given value of $B_{1}$ and $B_{2}$ can then be calculated as $[20]$

$$
I\left(B_{1}, B_{2}\right)=\sum_{v} P_{v} \sum_{f} \sum_{i}\left\langle\psi_{f i} \mid \psi_{f i}\right\rangle
$$

where

$$
\left|\psi_{f i}\right\rangle=\sqrt{P_{\text {hex } 2}(f)} U\left(B_{2}\right) R\left(\theta_{2}\right) S R\left(\theta_{1}\right) U\left(B_{1}\right) \sqrt{P_{\text {hex } 1}(i)}\left|\psi_{i}\right\rangle
$$

The sums run over the nine initial $m_{I}, m_{J}$ states $i$, the nine final $m_{I}, m_{J}$ states $f$ and the velocity $v$. The sum over the velocity is weighted by the contribution each velocity makes to the signal, $P_{v}$, which is modelled as a gaussian centred at $1444 \mathrm{~m} / \mathrm{s}$ with a full width at half maximum of $4 \%$ (approx. $58 \mathrm{~m} / \mathrm{s}$ ). The first and second rotation matrices $R(\theta)$ change the quantisation axis direction from $z$ to the surface normal, and from the surface normal to $z^{\prime}$ respectively.

It is important to note that two terms in eqn (2), $U\left(B_{1}\right)$ and $U\left(B_{2}\right)$, are particularly sensitive to the velocity, changing substantially between molecules with time of flights which 
differ by micro seconds. Thus we expect the signal to rise above or fall below it's average value, only for particular magnetic field values where this velocity dependence vanishes, in analogy to the standard spin echo condition.

Examples of signals that have been calculated scanning $B_{1}$ for a fixed value of $B_{2}=$ 545 gauss $\cdot \mathrm{m}$ are presented in Fig. 3. Whilst it would be desirable to look at examples of calculated scattering matrices for this particular system to compare to the experimental data, obtaining such matrices is challenging for theoretical methods [21], meaning few estimates of $S$ matrices are currently available. Instead, we have chosen to simulate the signals for a few different $S$ matrices to provide examples of the features in the spin echo signal that characterise the underlying $S$ matrix; the values used can be found in the Supplementary Information. Panel a shows the signal calculated for an identity scattering matrix, where the collision with the surface does not change the $m_{J}$ state of the molecule, and panel $\mathrm{b}$ the signal for a random but unitary scattering matrix where the collision can change the $m_{J}$ state of the $\mathrm{H}_{2}$ molecule, but all of the molecules scatter into the specular channel. The signals in panels c and d were calculated using two different random non-unitary scattering matrices, which are more representative of a realistic scattering scenario as they represent the case where molecules can be scattered into different channels (e.g., either specular or diffraction). Similarly to what we observed in our experiments, each of the calculated signals contain multiple echoes in addition to the standard spin echo condition $\left(B_{1}=545\right.$ gauss $\left.\cdot \mathrm{m}\right)$. Another observation is the complexity of the signal and, while there are some similarities, the signals corresponding to different scattering matrices are very different, demonstrating the dependence of the intensity of the echo signals on the scattering matrix elements.

Despite the wealth of information that these signals contain, only a small fraction of the possible echo conditions are encountered in these measurements due to the fixed value of $B_{2}$. In order to see a more complete picture both $B_{1}$ and $B_{2}$ need to be scanned. The complex signal obtained in such a $2 \mathrm{~d}$ scan is shown in Fig. 4. These calculations, which scan the magnetic fields $B_{1}$ and $B_{2}$ in the range of -600 gauss $\cdot m$ to 600 gauss $\cdot m$ with a resolution of 1 gauss.m contain 360000 values and require some post processing to be able to present the data in an accessible way. We chose to plot the value of the signal after dividing by the mean and then shifting the data so the mean is zero. Presenting the data in this way highlights the areas of the $B_{1}, B_{2}$ parameter space where there are echoes, whereas values close to zero (coloured white) represent regions where the different velocities lead to 

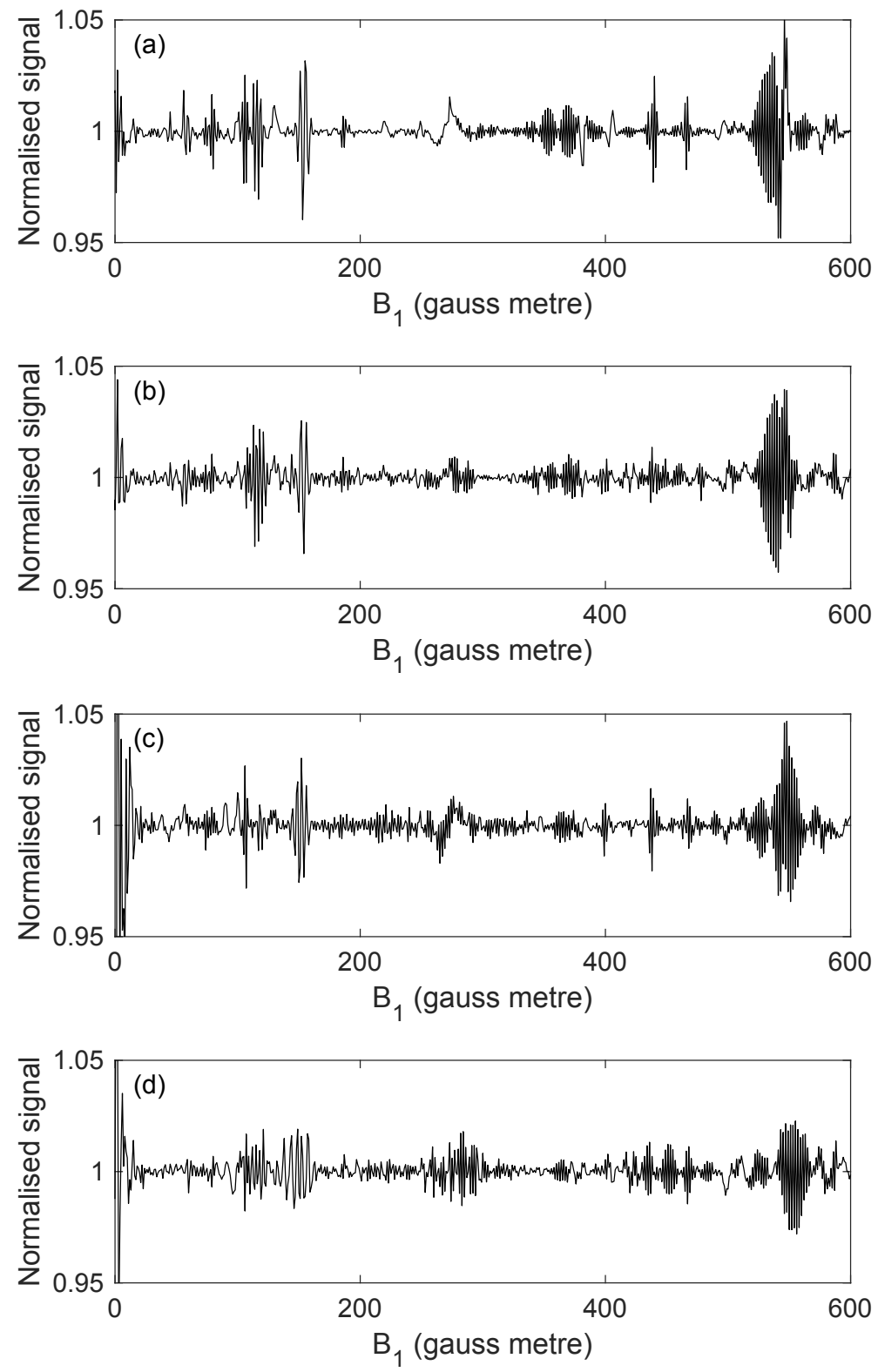

FIG. 3: Calculated signals for $B_{2}=545$ gauss.m using (a) an identity scattering matrix, (b) a unitary scattering matrix, and (c) and (d) two random scattering matrices.

destructive interference and the signal oscillations vanish.

These four very different $2 \mathrm{~d}$ images illustrate the true complexity of the multiple echo phenomena in this nine-level quantum system and the many different conditions where partial coherency can be regained. Most of the features will not have any simple interpretation and represent the generally quite complicated evaluation of eqn (2) for a particular $S$ matrix, i.e., for a particular molecule-surface interaction potential and specific scattering conditions. 

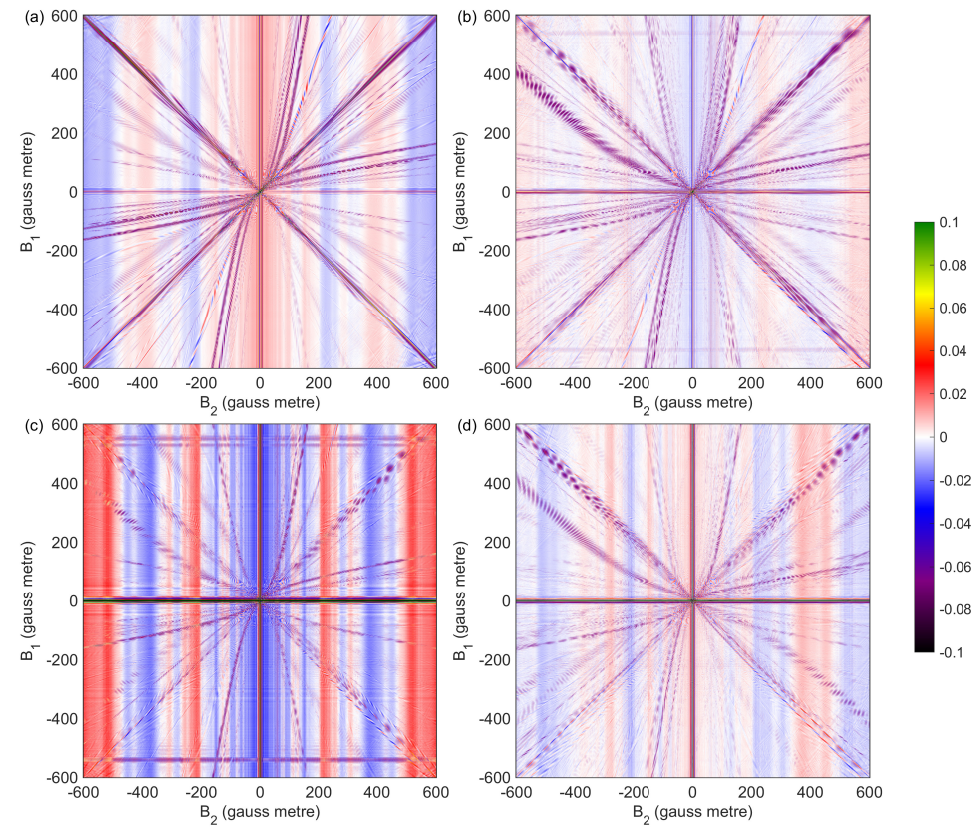

FIG. 4: Calculated signals changing both $B_{1}$ and $B_{2}$ using (a) an identity scattering matrix, (b) a unitary scattering matrix, and (c) and (d) two random scattering matrices. The same scattering matrices were used as for Fig. 3 .

In contrast, some features can be understood more intuitively. For example, the $45^{\circ}$ and $-45^{\circ}$ diagonal lines along the $B_{1}=B_{2}$ and $B_{1}=-B_{2}$ conditions are analogous to the simple parallel and anti-parallel echoes you would also expect in the spin $\frac{1}{2}$ ( 2 level) system. The many other diagonal features could arise from the many different frequencies the signal is comprised of in this nine-level system: Crudely speaking, if the gyromagnetic ratio for one transition was exactly double another then this would be expected to give lines at $B_{1}=$ $\left|2 B_{2}\right|$ and $B_{2}=\left|2 B_{1}\right|$. The curvature of the lines at low magnetic fields highlight the field independent spin-rotation and spin-spin coupling terms in the Hamiltonian, which become less significant at higher fields where the echoes fall on straighter cuts through the $B_{1}, B_{2}$ parameter space.

Another interesting feature which has a relatively simple interpretation is the strong horizontal line which appears in the calculations for a general non-unitary matrix (panels c and d) around $B_{1}=0$ gauss $\cdot \mathrm{m}$. For a non-unitary matrix, the probability of scattering into the specular channel changes as a function of the quantum state of the molecules when they arrive at the surface. For non-magnetic molecule-surface interactions this would be expected 
to be related to the rotational states rather than the nuclear spin states. Thus, when we scan $B_{1}$ and continuously change the rotational state of the molecules arriving at the sample, the flux of the scattered molecules will change, producing oscillations in the signal even in the absence of quantum state selection in the second arm of the apparatus. As $B_{1}$ is increased further away from $B_{1}=0$ gauss $\cdot \mathrm{m}$, the difference in the quantum state of molecules arriving at the sample with different velocities will become greater and these oscillations will decay due to the resulting loss of coherency. This can be seen in Fig. 5a, where the red dashed line represents the total flux entering the second arm, summing over all the different quantum states. We will refer to this as a flux-detection measurement which is what we would expect to measure if the scattering arm of the instrument (illustrated schematically in Fig. 1) would not include the analyser magnet, and any molecule leaving the sample in the direction of the particle detector would be detected regardless of its quantum state. In this case, the scattered intensity, $I\left(B_{1}\right)$ is given by an analogous expression to eqn (1), where the relevant wave-function, $\left|\psi_{f i}^{\prime}\right\rangle$ is that immediately after the scattering event

$$
\left|\psi_{f i}^{\prime}\right\rangle=S R\left(\theta_{1}\right) U\left(B_{1}\right) \sqrt{P_{\text {hex } 1}(i)}\left|\psi_{i}\right\rangle
$$

Figure $5 \mathrm{~b}$, which focusses on the region around $B_{1}=0$ gauss $\cdot \mathrm{m}$, shows the close resemblance between a solution of eqn (2) calculated at a value of $B_{2}=545$ gauss.m (black line) and that expected in a flux-detection measurement given by eqn (3) (red dashed line). Figs $5 \mathrm{c}$ and $5 \mathrm{~d}$ show the same comparison for the random non-unitary scattering matrix used to generate Fig. 4d. In this case there is still quite a high correlation between the two but there are also notable differences, emphasising that scanning $B_{1}$ while maintaining $B_{2}$ at some high value is often a good approximation of a flux-detection signal, but may also contain further contributions to the signal and requires a full solution of eqn (2) to be calculated accurately. The strong oscillations observed experimentally near $B_{1}=0$ gauss $\cdot \mathrm{m}$ (shown in Fig. 2e), are an experimental demonstration of this phenomena.

There is also another faint but distinguishable horizontal line at approximately $B_{1}=$ \pm 540 gauss $\cdot \mathrm{m}$ which appears in some of the $2 \mathrm{~d}$ plots shown in Fig. 4 . A hint to the origin of this line can be seen by looking at a calculation of the rotational populations of the molecules, as a function of $B_{1}$, when they arrive at the surface, as shown in Fig. 6a. Initially strong oscillations are seen as a function of $B_{1}$, leading to the flux-detection oscillations mentioned earlier. However as the field is increased, the average over the different 

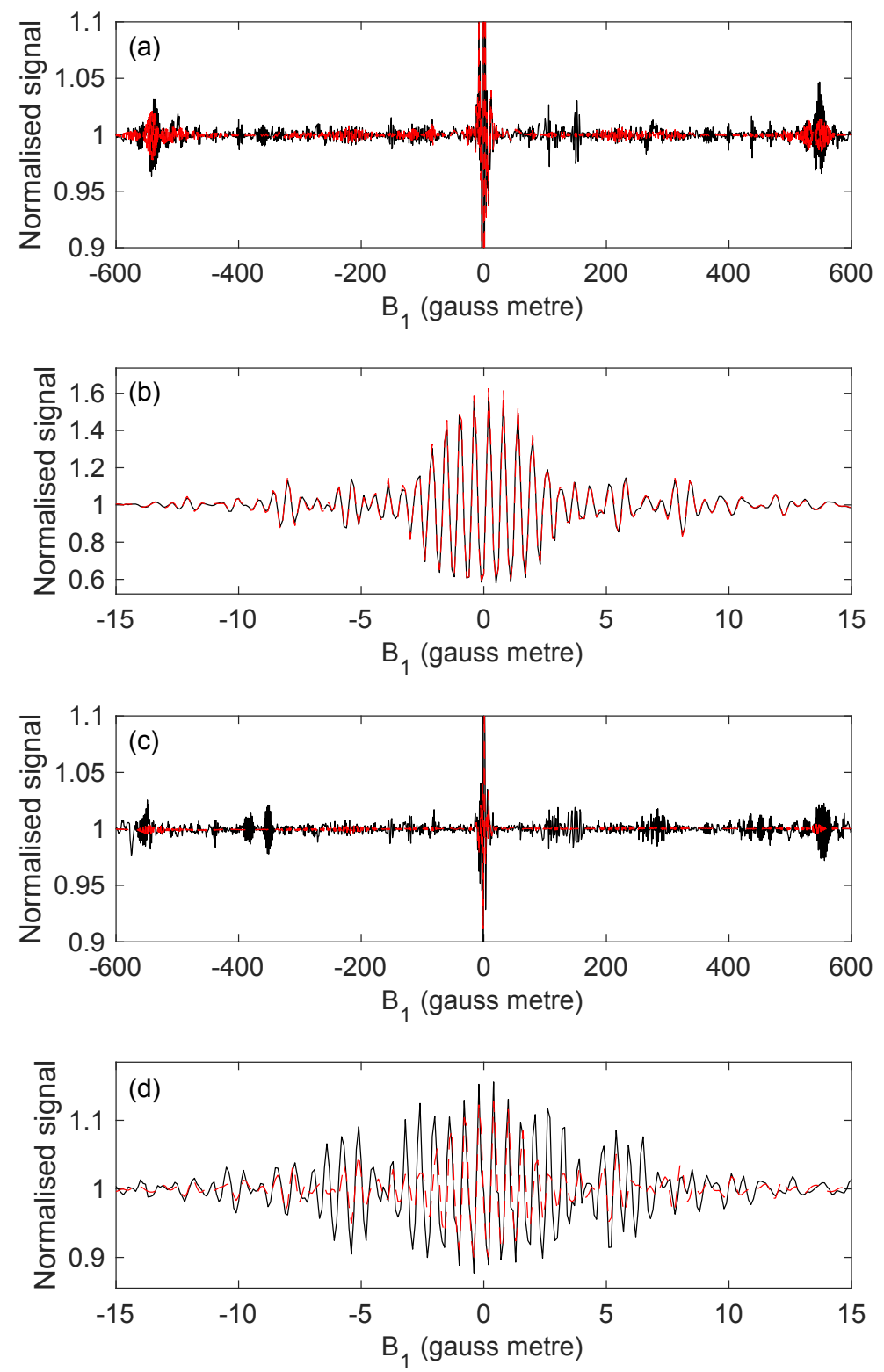

FIG. 5: Panels (a) and (c) show a comparison of the full signal (black) calculated at a value of $B_{2}$ $=545$ gauss $\cdot \mathrm{m}$ which corresponds to a slice through Figs. 4c and $4 \mathrm{~d}$ and the flux-detection signal (red dashed line) that would be obtained without the analyser hexapole. Panels (b) and (d) are a magnification of panels (a) and (c) around $B_{1}=0$ gauss $\cdot \mathrm{m}$ respectively.

velocities gradually reduces these oscillations due to the loss of coherency. At a field value of approximately \pm 540 gauss $\cdot \mathrm{m}$, oscillations in the rotational populations can again be seen. The position of this rotational focussing effect depends on both the magnetic field dependent and the magnetic field independent terms in the Hamiltonian and correspondingly changes 

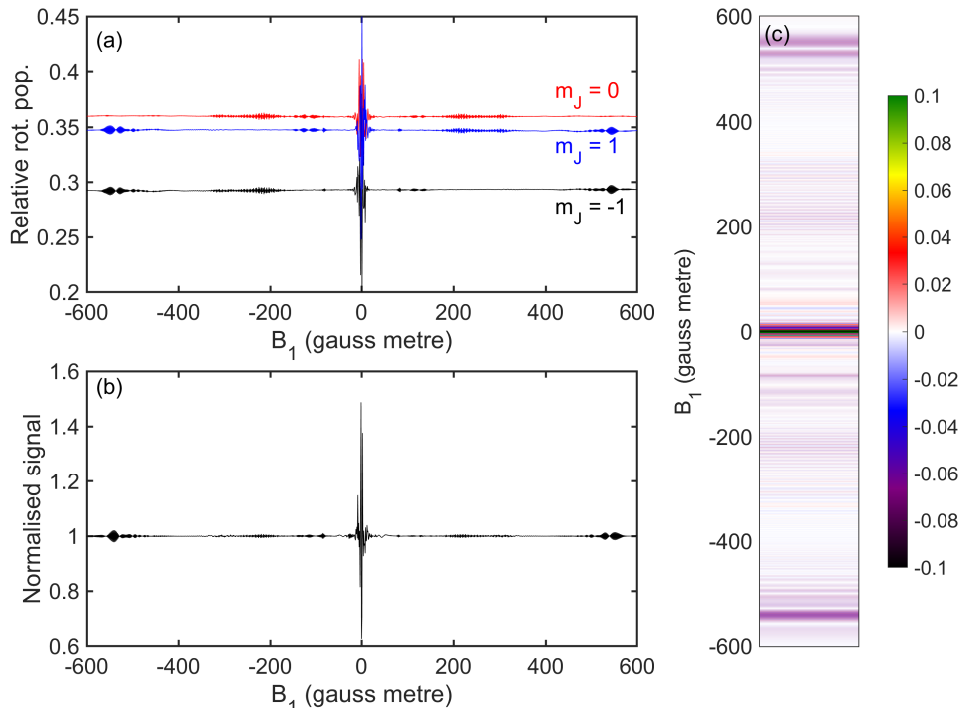

FIG. 6: The change of the $m_{J}=-1$ (black), $m_{J}=0$ (red) and $m_{J}=1$ (blue) populations calculated at a velocity of $1444 \mathrm{~m} / \mathrm{s}$ as a function of $B_{1}$ where the surface normal is taken as the quantisation axis (a), and an example of a flux-detection calculated signal (b). Panel (c) shows the same fluxdetection signal presented in the same way as Fig. 4 to aid with the comparison of the signals. The scattering matrix used to generate panels (b) and (c) is the same as that used in Figs. 3c and $4 \mathrm{c}$

for different magnetic field profiles and flight times through the first arm. Figure 7 compares the rotational populations at the surface (panel a) and the resulting flux-detection signal (panels b and c) for a mean velocity of $2000 \mathrm{~m} / \mathrm{s}$ and shows the correspondence between the two. In particular, at about $B_{1}=300$ gauss $\cdot \mathrm{m}$ changes in the $m_{J}$ state populations are seen which is also reflected in a high intensity region in the corresponding flux-detection simulation.

Finally, there are also strong vertical lines in the $2 \mathrm{~d}$ maps shown in Fig. 4, which correspond to features which are independent of $B_{1}$. These features are observed when there are differences in the $m_{J}$ state populations that scatter from the surface which then evolve differently for different values of $B_{2}$. This gives rise to an oscillation due to the analysing hexapole. In the case of the identity matrix in Fig. 4a where all the states scatter with the same probabilities, the oscillations can be attributed to the differences in the $m_{J}$ state 

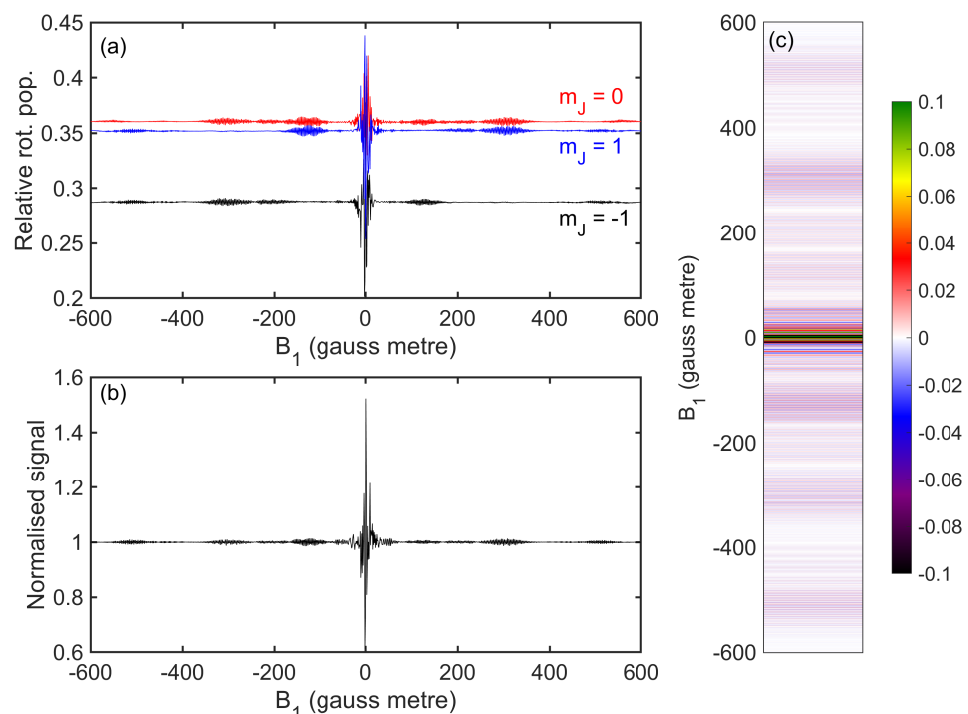

FIG. 7: Same as Fig. 6, but for a velocity of $2000 \mathrm{~m} / \mathrm{s}$.

populations hitting the surface (see Fig. 6a). For more general scattering matrices, where the probability of scattering into the different states is likely to be different, the vertical stripes would be seen even if equal populations of the $m_{J}$ states collide with the surface, as the different scattering probabilities would result in different $m_{J}$ state populations in the scattered molecular beam. Experimentally, we would expect to be sensitive to only these vertical features if we removed the polariser hexapole from the first arm of the machine which would result in equal populations of the nine $m_{I}, m_{J}$ states hitting the surface, irrespective of the value of $B_{1}$. The results from a simulation where the polariser probabilities are all equal is shown in Fig. 8, where panel a corresponds to the scattering matrix used to produce Fig. 4c, and panel b the scattering matrix for Fig. 4d. The oscillations are stronger in Fig. 8a because the collision with the surface creates a larger difference in the $m_{J}$ state populations after scattering than in Fig. 8b.

To demonstrate that the features described above are general features of the $2 \mathrm{~d}$ signals rather than specific to the $S$ matrices that we have chosen, Fig. 9 shows the total signal obtained from calculations using 544 random non-unitary $S$ matrices. Here, each individual signal has been normalised to it's mean and then shifted before the absolute value was summed. Adding the absolute signals in this way allows the regions of the $B_{1}, B_{2}$ parameter space where there are echoes to be clearly distinguished, whereas just adding the signals could 


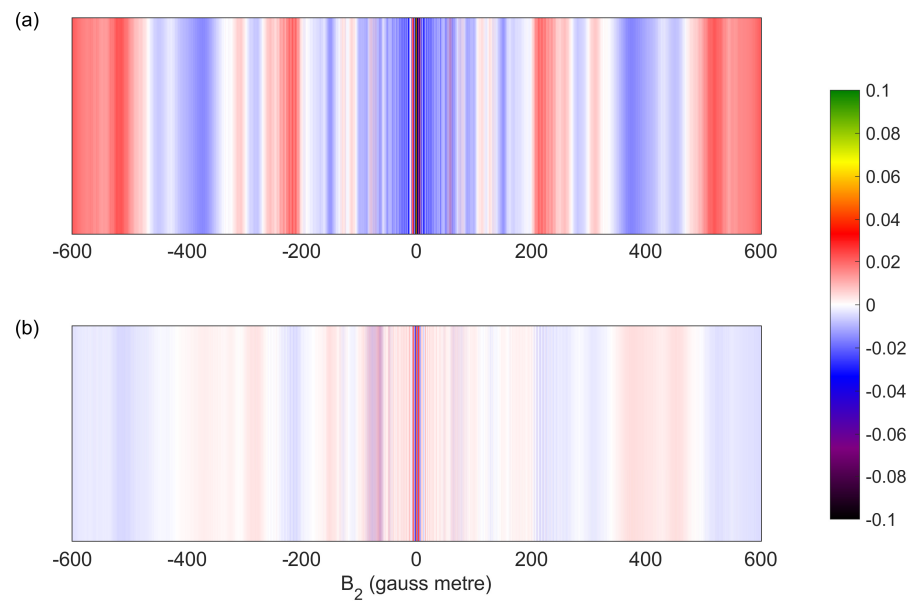

FIG. 8: Simulated signals for the two random scattering matrices used throughout when the probability of the nine $m_{I}, m_{J}$ states passing through the polarising hexapole are all equal.

result in the echoes destructively interfering and summing to give zero. As can be seen, there are regions of the $B_{1}, B_{2}$ parameter space where echoes could be measured and other regions where there are none, which can be attributed to the discrete energy differences between the nine $m_{I}, m_{J}$ states at a given value of magnetic field. To maximise the information that can be obtained about the underlying $S$ matrix, future experiments should focus on measuring the intensity of scattered molecules in these regions. The features which have been described above including the flux-detection features around $B_{1}=0$ gauss $\cdot \mathrm{m}$ and $B_{1} \approx \pm 540$ gauss $\cdot \mathrm{m}$ and the vertical stripes are also seen. As has been shown, it is the relative intensity of each of these features, as well as the diagonal echoes, which depends sensitively on the underlying $S$ matrix.

\section{CONCLUSIONS AND OUTLOOK}

The existence of multiple coherences in magnetically-manipulated molecular beam experiments has been demonstrated empirically and theoretically. The multiple echo patterns that are seen in the scattered intensity provide a fingerprint, characteristic of the scattering matrix, which contains information about the molecule-surface interaction potential. Being able to perform these measurements experimentally opens the possibility of obtaining 


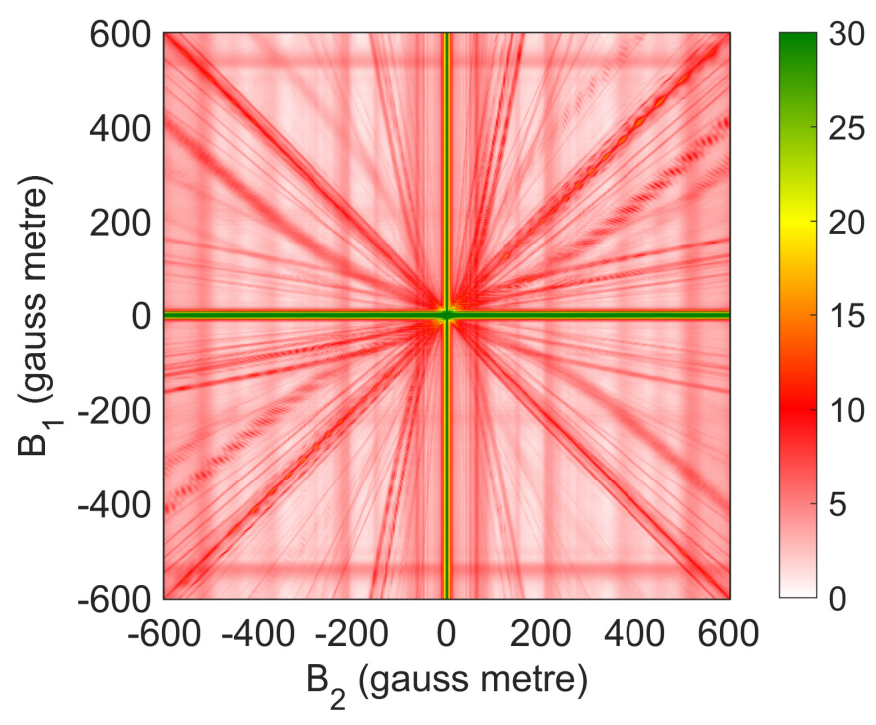

FIG. 9: The sum of the absolute signals that have been simulated using 544 random non-unitary scattering matrices.

empirically determined scattering matrices by fitting the experimental data. This should be possible as in the case of the experiments, the only unknown in eqns (1) and (2) is $S$. Repeating experiments over a large number of $B_{1}$ and $B_{2}$ values can provide enough information to allow the scattering matrix to be extracted from the data, similarly to what has been achieved with 1d data measured for diffractive scattering [20]. Applying new machine learning algorithms, for example Gaussian Process models [22], could potentially reduce the number of $B_{1}$ and $B_{2}$ values that would be required to obtain an empirical scattering matrix in this way. The scattering matrices that we extract not only provide a new and stringent benchmark which will help guide the development of accurate theoretical models of gassurface collisions, but also provide $m_{J}$ state to $m_{J}$ state scattering probabilities, which in turn can be used to determine to what extent collisions of $\mathrm{H}_{2}$ with the surface rotationally polarise the scattered molecular beam. The accuracy to which we can determine the scattering matrix is limited by the width of the velocity distribution, as the scattering matrix will change as the energy of the incident $\mathrm{H}_{2}$ molecules changes. Consequently as the width of the distribution becomes wider more energies will contribute to the signal, so we would, in effect, extract a scattering matrix which was averaged over the collision energy.

While time consuming, mapping a large fraction of $B_{1}, B_{2}$ space will be particularly beneficial when measurements can not be performed at diffraction conditions, e.g. on smooth 
surfaces where only specular scattering produces a measurable signal. For specular scattering the energy distribution width leads to a fast decay of the measurable oscillations, and measuring the intensity of the various echoes, where coherency is regained, and comparing the measurements with calculations using eqns (1) and (2) presents an alternative and very sensitive way of assessing the molecule-surface interaction.

Finally, the analysis presented in this paper also serves as an important guide for future attempts to perform molecular spin echo experiments of surface dynamics. One motivation for performing dynamics measurements with molecules, rather than atoms, is the potential ability to measure rotational motion on surfaces. While helium spin echo is particularly well suited to measure translational motion on surfaces, rotation of molecules on surfaces could not be directly measured with helium spin echo even when the rate of motion implies that such rotations are the dominant motion [23]. The fact that a molecular beam probe can exchange both rotational and translational momentum, could potentially make it possible to measure surface rotations directly. An encouraging observation in this aspect is that $m_{J}$ flips allow a hydrogen beam to detect phonons which can not be seen in helium scattering [14]. However, there are significant complications which need to be taken into account when planning such experiments. First, the calculations presented in this work are limited to scattering events which do not depend on the arrival time of different wave packets corresponding to different quantum states, i.e., a static surface. To account for the dynamics a different level of theory would be needed where both the magnetic Hamiltonian and the centre of mass motion of the molecules are treated quantum mechanically [24]. Nevertheless, the scattering calculations presented above already show that along a particular spin echo condition (for example $B_{1}=B_{2}$ ) the intensity changes due to the many different refocussing possibilities of a nine-level quantum system even when the surface is static. In a typical spin $\frac{1}{2}$ measurement of surface dynamics, the intensity along the spin echo condition line would remain constant, and any decay as a function of the magnetic field magnitude would be related to motion on the surface [5]. Consequently, a more complicated analysis, which includes both the interference conditions of the multiple echoes due to static scattering, as well as the loss of coherency due to surface motion, will be required for extracting the dynamics from a molecular spin echo experiment. 


\section{Conflicts of interest}

There are no conflicts to declare.

\section{Acknowledgements}

This work was funded by an ERC consolidator grant (Horizon 2020 Research and Innovation Programme grant 772228).

[1] F. Mezei, Zeitschrift für Physik A Hadrons and Nuclei, 1972, 255, 146-160.

[2] F. Mezei, The principles of neutron spin echo, Springer Berlin / Heidelberg, 1980, vol. 128, pp. $1-26$.

[3] Neutron Spin Echo Spectroscopy, ed. C. P. F. Mezei and T. Gutberlet, Springer-Verlag Berlin Heidelberg, 2003.

[4] M. DeKieviet, D. Dubbers, C. Schmidt, D. Scholz and U. Spinola, Physical Review Letters, 1995, 75, 1919-1922.

[5] A. Jardine, H. Hedgeland, G. Alexandrowicz, W. Allison and J. Ellis, Progress in Surface Science, 2009, 84, 323-379.

[6] G. Benedek and J. P. Toennies, Atomic Scale Dynamics at Surfaces, Springer, 2018.

[7] M. H. Levitt, Spin dynamics: basics of nuclear magnetic resonance, John Wiley \& Sons, 2013.

[8] I. Litvin, Y. Alkoby, O. Godsi, G. Alexandrowicz and T. Maniv, Results in Physics, 2019, 12, 381-391.

[9] L. Van Hove, Physical Review, 1954, 95, 249.

[10] R. Gähler, R. Golub, K. Habicht, T. Keller and J. Felber, Physica B: Condensed Matter, 1996, 229, $1-17$.

[11] G. Alexandrowicz and A. P. Jardine, J. Phys.: Cond. Matt., 2007, 19, 305001.

[12] D. Farias and K.-H. Rieder, Reports on Progress in Physics, 1998, 61, 1575-1664.

[13] C.-f. Yu, K. B. Whaley, C. S. Hogg and S. J. Sibener, Physical review letters, 1983, 51, 2210.

[14] G. Benedek, F. Traeger and J. P. Toennies, Phys. Rev. Lett., 2005, 94, 086103.

[15] N. F. Ramsey, Physical Review, 1952, 85, 60. 
[16] O. Godsi, G. Corem, Y. Alkoby, J. T. Cantin, R. V. Krems, M. F. Somers, J. Meyer, G.-J. Kroes, T. Maniv and G. Alexandrowicz, Nature communications, 2017, 8, 15357.

[17] A. P. Jardine, P. Fouquet, J. Ellis and W. Allison, Review of Scientific Instruments, 2001, 72, $3834-3841$.

[18] M. Utz, M. H. Levitt, N. Cooper and H. Ulbricht, Phys. Chem. Chem. Phys., 2015, 17, $3867-3872$

[19] S. Dworski, G. Alexandrowicz, P. Fouquet, A. P. Jardine, W. Allison and J. Ellis, Review of Scientific Instruments, 2004, 75, 1963-1970.

[20] Y. Alkoby, H. Chadwick, O. Godsi, H. Labiad, M. Bergin, J. T. Cantin, I. Litvin, T. Maniv and G. Alexandrowicz, Nature Communications, 2020, 11, 3110.

[21] R. C. Mowrey and G. J. Kroes, The Journal of Chemical Physics, 1995, 103, 1216-1225.

[22] C. E. Rasmussen and C. K. I. Williams, Gaussian Processes for Machine Learning, MIT Press, Cambridge, 2006.

[23] H. Hedgeland, M. Sacchi, P. Singh, A. J. McIntosh, A. P. Jardine, G. Alexandrowicz, D. J. Ward, S. J. Jenkins, W. Allison and J. Ellis, The Journal of Physical Chemistry Letters, 2016, 7, 4819-4824.

[24] J. T. Cantin, G. Alexandrowicz and R. V. Krems, Phys. Rev. A, 2020, 101, 062703. 